Recent Insights into the Physics of the Sun and Heliosphere:

Highlights from $\mathrm{SOHO}$ and Other Space Missions

IAU Symposium, Vol. 203, 2001

P. Brekke, B. Fleck, and J. B. Gurman eds.

\title{
Fractional Frequency Shifts of Local Helioseismic Modes With Magnetic Activity Using Ring-Diagram Analysis
}

\author{
B. Hindman, D. Haber, J. Toomre
}

JILA and Dept. of Astrophysical and Planetary Sciences, University of Colorado, Boulder, CO 80309-0440, USA

\author{
R. S. Bogart
}

Stanford University, HEPL-CSSA, Stanford, CA 94305-4085, USA

\begin{abstract}
Using full-disk Doppler velocity data from MDI on SOHO, we have determined the central frequencies of high-degree $\mathrm{p}$ modes and $f$ modes over a dense mosaic of localized regions on the sun using ringdiagram analyses (Haber et al. 2000). The motion of active regions as they rotate across the solar disk is well traced by changes in the central frequencies of the acoustic wave modes. Active regions appear as locations of large positive frequency shifts.
\end{abstract}

\section{Introduction}

The frequencies of solar acoustic oscillations are clearly sensitive to the solar cycle. As the solar cycle advances and magnetic activity rises, the frequencies increase (Libbrecht \& Woodard 1990). Howe, Komm, \& Hill (1999) reported that the latitudinal distribution of the perturbation in frequencies is well matched by the latitudinal distribution of the surface magnetic field. Due to the symmetries of the modes used, such global analyses are incapable of probing the latitudinal asymmetries or longitudinal variation. Local helioseismic techniques, such as ring-diagram analyses, lack these restrictions and have the capability to completely specify the spatial distribution of the perturbation.

\section{Variation of Mode Central Frequencies}

Cuts at constant frequency of the 3-D power spectra of a sequence of Doppler images reveal a nested set of rings in power, each ring corresponding to a different radial order of the acoustic wave field. The ring-diagram analyses fit the power spectra with a prescribed functional form (Haber et al. 1998). This fitting function contains six parameters: the central frequency of the mode, the mode width, the mode amplitude, the background amplitude, and two ring displacements associated with horizontal flows. Through this fitting the central frequencies are determined as a function of harmonic degree $\ell$ and mode order $n$ for a given localized region or tile. Using MDI data on a daily basis during the Dynamics Programs, we repeat the analysis over a dense-pack mosaic of overlapping tiles (Haber et al. 2000). The frequencies are thereby determined as a function of the date and position on the solar disk. Figure 1 displays the resulting frequencies for a single 1664-minute period starting on 4 April 1999. A careful examination of a series of images of this type for different days reveals that the variation of mode central frequencies with position on the solar disk 
involves two components: a stationary component due to instrumental effects and another associated with solar activity that rotates with the solar surface.

The stationary component of the frequency variation is computed by averaging the frequency at a given disk location in time using only those dense-pack tiles in which the rms field strength (as determined by MDI magnetograms) is less than $20 \mathrm{G}$. The spatial variability in the stationary component is the result of image scale variations across the MDI field-of-view. From tile to tile the local wavenumber is thus mismeasured and an incorrect mode frequency is attributed to a given wavenumber or harmonic degree. For the scientifically more interesting variations, we calculate the fractional frequency shift associated with surface magnetism by subtracting the stationary frequency component from the local mode frequencies. Figure 2 presents a series of images of the fractional frequency shift for the $n=4, \ell=500$ mode from consecutive 1664-minute periods. Each is paired with a coeval magnetogram. Active regions consistently appear as regions of large positive fractional frequency shift.

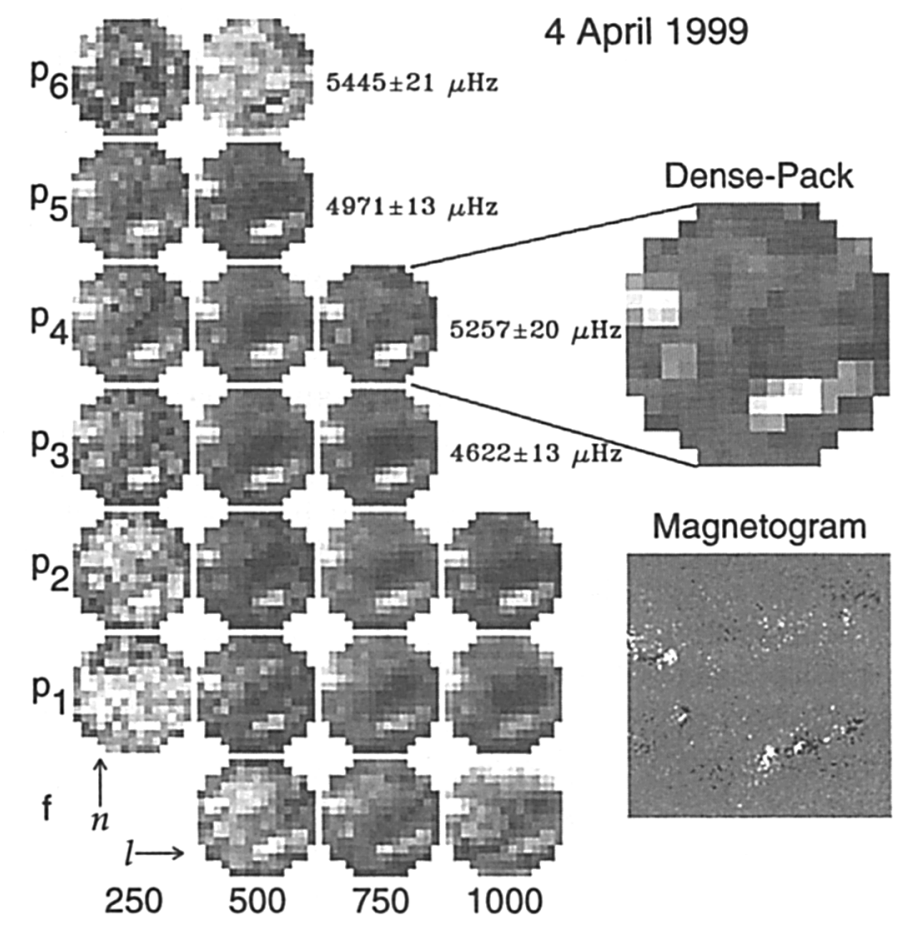

Figure 1. Maps of mode central frequencies with position on solar disk on 4 April 1999 as determined by ring-diagram analyses of the dense-pack data set. Each of the smaller images corresponds to a different radial order $n$ and harmonic degree $\ell$. The large image at topright is an enlargement for the $n=4, \ell=750$ mode for the mosaic of sites on the disk. The magnetogram spans the same region of the solar surface covered by the dense-pack mosaic, and is coeval with the Doppler observations. The local frequency changes strikingly across the disk, varying as much as $40 \mu \mathrm{Hz}$ for some modes as indicated. 


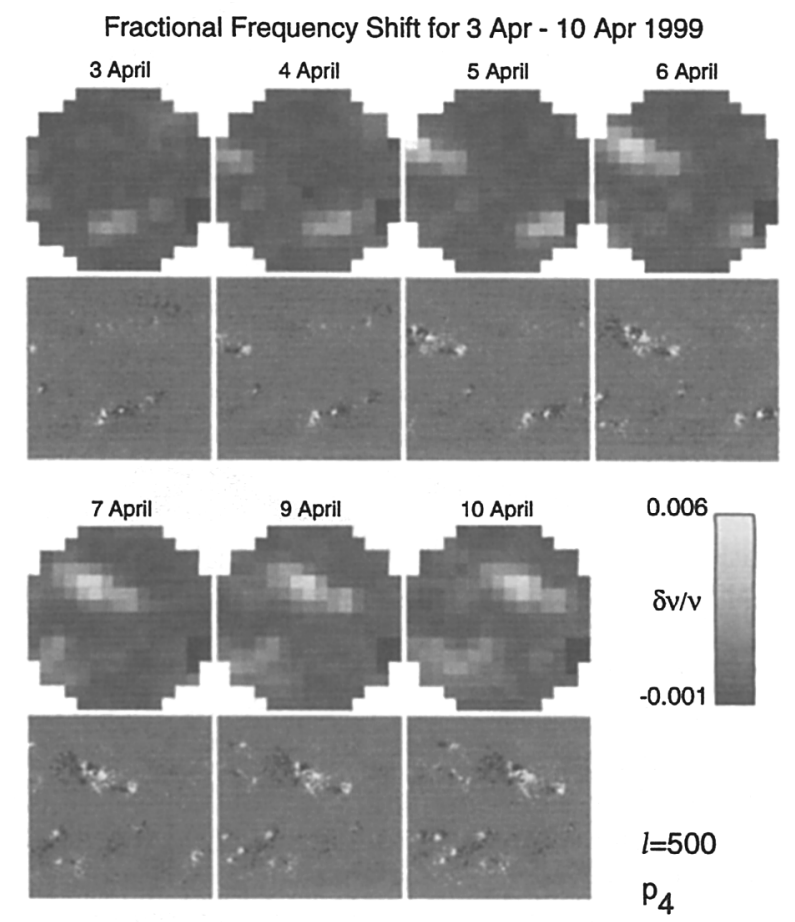

Figure 2. A sequence of images of the fractional frequency shift with position on solar disk determined on successive days for the $n=4$ and $\ell=500$ wave modes, paired with coeval magnetograms. The passage of active regions across the solar disk as solar rotation carries them from left to right is clearly traced by the frequency shifts. Within active regions the frequency shifts are always positive.

\section{Local Mode Frequencies and Magnetism}

To assess the links between frequency shifts and magnetism at a given site, we average all tiles (in position and time) which have an rms magnetic field strength within certain predefined ranges. This allows us to measure the frequency shift as a function of activity. Figure 3 shows the average frequency shift for 1999 for two different ranges of field strength. The general rise in frequency shift with frequency, independent of radial order, is indicative of a perturbation located at the solar surface. The modulation of the low-order mode curves for the highest field strengths corresponds to a perturbation of the acoustic cavity itself.

The frequency dependence of the local frequency shifts averaged over the dense-pack mosaic strongly resembles the shifts of global mode frequencies that occur over the solar cycle (Libbrecht \& Woodard 1990; Howe, Komm, \& Hill 1999). It is likely that the high-degree and low-degree shifts are produced by the same phenomena, namely, surface magnetism, particularly in the form of active regions. As with the global shifts, the local frequency shifts are predominantly 


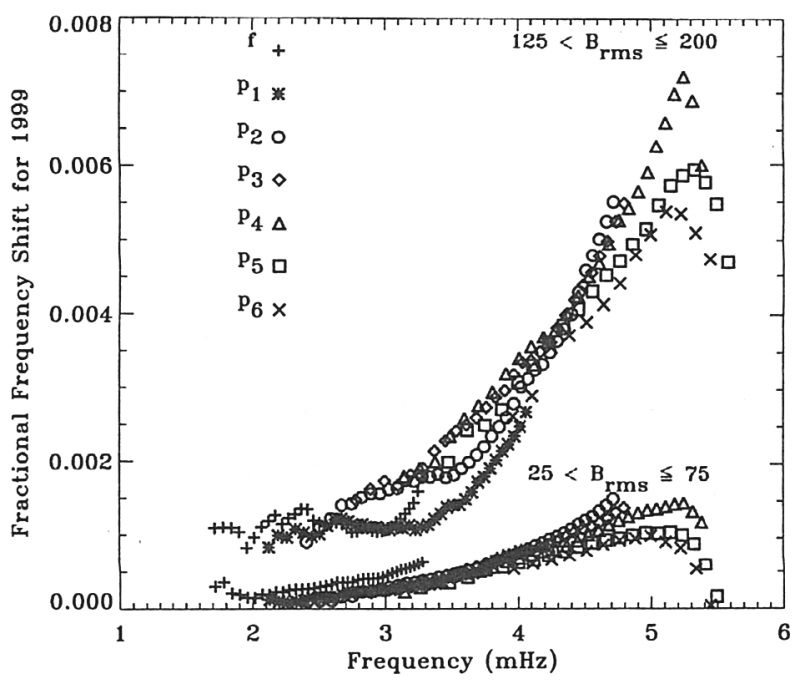

Figure 3. The fractional frequency shifts as a function of frequency averaged over tiles in 1999 that have an rms magnetic field strength within both low and high ranges as indicated. The general increase in frequency shift as a function of frequency is indicative of a perturbation which is located at the solar surface. The modulation evident in the low-order mode curves for the highest field strengths suggests that a perturbation of the acoustic cavity occurs in such regions.

a function of frequency. This is indicative of a perturbation of the surface reflection conditions of the acoustic waves. For the strong magnetic field strengths, the local frequency shift evinces a variation with harmonic degree $\ell$ as well as frequency. This behavior is consistent with a sound speed perturbation within the acoustic cavity. In the future, we hope to determine the local sound speed perturbation below active regions by inverting these frequency shifts.

Acknowledgments. This research was supported by NASA through grants NAG 5-8133 and NAG 5-7996 and by NSF through grant ATM-9731676. The SOI-MDI project is supported by NASA grant NAG 5-3077 to Stanford University. SOHO is a project of international cooperation between ESA and NASA.

\section{References}

Haber, D. A. et al. 1998, in SOHO6/GONG 98 Workshop, ed. S. Korzennik and A. Wilson, (Noordwijk: ESA), 791

Haber, D. A., Hindman, B. W., Toomre, J., and Bogart, R. S. 2000, Solar Phys., 192,363

Howe, R., Komm, R., and Hill, F. 1999, ApJ, 524, 1084

Libbrecht, K. G. and Woodard, M. F. 1990, Nature, 345, 779 